\title{
IMPLANTAÇÃO DE FLUXOGRAMA DE ATENDIMENTO EM UM CENTRO DE ATENÇÃO PSICOSSOCIAL
}

\section{Implementation of a Healthcare Workflow in a Psychosocial Care Center \\ Implantación del flujo de trabajo de la atención de un centro de atención psicosocial}

\author{
Maria do Socorro Távora de Aquino \\ Universidade da Integração Internacional da Lusofonia Afro - Brasileira - UNILAB - Acarape (CE) - Brasil \\ Pedro Holanda Souza \\ Universidade da Integração Internacional da Lusofonia Afro - Brasileira - UNILAB - Acarape (CE) - Brasil
}

Francisco Clécio da Silva Dutra

Universidade da Integração Internacional da Lusofonia Afro - Brasileira - UNILAB - Acarape (CE) - Brasil

\section{Patrícia Freire de Vasconcelos}

Universidade da Integração Internacional da Lusofonia Afro - Brasileira - UNILAB - Acarape (CE) - Brasil

\section{RESUMO}

Objetivo: Relatar a implantação de um fluxograma para os atendimentos de rotina em um Centro de Atenção Psicossocial (CAPS). Síntese dos dados: Relato de experiência de caráter intervencionista desenvolvido por acadêmicos do curso de Enfermagem, do Instituto de Ciências da Saúde, vinculado à Universidade da Integração Internacional da Lusofonia Afro-Brasileira (UNILAB), no município de Redenção-Ceará BR. A elaboração do fluxograma iniciou-se no mês de abril de 2016 e foi realizada mediante observação sistemática do processo de trabalho da equipe de saúde do CAPS. Buscou-se criar um ambiente acolhedor, com escuta qualificada na chegada das pessoas usuárias, orientandoas sobre como e onde seria seu atendimento para minimizar dúvidas. Enquanto aguardavam atendimento, realizaram-se rodas de conversa com discussão de diversos temas sugeridos pelos usuários. A partir das observações participantes e das rodas de conversa, foi criado o novo fluxograma com apoio dos profissionais e gestores do serviço. Constatou-se a importância da implantação de um fluxograma para a melhoria na organização do processo de trabalho da unidade. Conclusão: Prover estratégias que proporcionem acolhimento aos usuários dos serviços de saúde, principalmente nos CAPS, se torna uma ferramenta indispensável para a execução de um tratamento eficaz e eficiente.

Descritores: Fluxo de Trabalho; Acolhimento; Centros de Atenção Psicossocial.

\section{ABSTRACT}

Objective: To report the implementation of a routine care workflow in a Psychosocial Care Center (Centro de Atenção Psicossocial - CAPS). Data synthesis: An experience report of an intervention carried out by Nursing students of the Health Sciences Institute of the the University for International Integration of the Afro-Brazilian Lusophony (Universidade da Integração Internacional da Lusofonia Afro-BrasileiraUNILAB) in the municipality of Redenção, Ceará, Brazil. The development of the workflow started in April 2016 through the systematic observation of the work process of the healthcare team in the CAPS. We sought to create a more welcoming environment by actively listening to incoming users and informing them about how and where they would be examined so as to minimize doubts. While waiting for care, users participated in group discussions about several issues suggested by themselves. Participant observation of the group discussions served as a basis for the development of a new workflow with the support from the center staff and managers. The implementation of a workflow for improving the organization of the work process in the center was of the utmost importance. Conclusion: Providing strategies to embrace health care users, particularly in the CAPS, is an indispensable tool for the delivery of an effective and efficient treatment.

Descriptors: Workflow; User Embracement; Psychosocial Care Centers. 


\section{RESUMEN}

Objetivo: Relatar la implantación de un flujo de trabajo para la asistencia de rutina de un Centro de Atención Psicosocial (CAPS). Síntesis de los datos: Relato de experiencia de intervención desarrollado por académicos del curso de Enfermería del Instituto de Ciencias de la Salud vinculado a la Universidad de la Integración Internacional de la Lusofonia Afro-Brasileira (UNILAB) en el municipio de RedençãoCeará - BR. La elaboración del flujo de trabajo se inició en el mes de abril de 2016 y se dio mediante la observación sistemática del proceso de trabajo del equipo de salud del CAPS. Se buscó crear un ambiente acogedor con una escucha de calidad en la llegada de los usuarios orientándolos sobre cómo y donde sería su consulta para minimizar las dudas. Mientras esperaban la consulta fueron realizadas ruedas de conversación con discusión de varios temas sugeridos por los usuarios. A partir de las observaciones participantes y de las ruedas de conversación se creó el nuevo flujo de trabajo con el apoyo de los profesionales y gestores del servicio. Se constató la importancia de la implantación de un flujo de trabajo para mejorar la organización del proceso de trabajo de la unidad. Conclusión: Estrategias que promocionen la acogida de los usuarios de los servicios de salud, principalmente en el CAPES es una herramienta indispensable para la ejecución de un tratamiento eficiente y eficaz.

Descriptores: Flujo de Trabajo; Acogimiento; Servicios de Salud Mental.

\section{INTRODUÇÃO}

No contexto brasileiro, foram muitas as conquistas no campo do cuidado à saúde mental. Entretanto, no que concerne à acessibilidade das pessoas usuárias ao atendimento, há obstáculos e desafios a superar decorrentes de fragilidades e barreiras apara sua resolutividade, sobretudo no que diz respeito ao funcionamento da própria instituição ${ }^{(1)}$.

O serviço de saúde mental sempre foi motivo de questionamentos devido à maneira como os pacientes eram tratados, suscitando sempre a questão da reforma psiquiátrica como uma estratégia de melhoria na atenção à saúde mental. Com a ampliação e humanização do serviço, com culminante abandono do modelo de internação manicomial, surge a reforma psiquiátrica no fim dos anos 70 , trazendo grandes avanços para o cuidado em saúde mental ${ }^{(2,3)}$.

A ética da reforma psiquiátrica fundamenta-se na defesa dos direitos civis dos pacientes, que antes não eram vistos com tais direitos. Visa um olhar sobre o comportamento, as atitudes e o modo de ser desses indivíduos, levando o profissional, a família e a comunidade a compreenderem seus valores. Implica em um novo lugar social para a loucura, permitindo o diálogo, tirando-os do eterno silêncio e recalque aos quais foram submetidos ${ }^{(4)}$.

Dentro dos diversos avanços ocorridos com a reforma psiquiátrica, pode-se citar os Centros de Atenção Psicossocial (CAPS), que visam dar suporte, tratamento e enfrentamento às doenças de cunho psiquiátrico e psicológico, fazendo com que os indivíduos com transtorno mental compreendam seu atual estado, bem como com que possam ser inseridos dentro do contexto familiar a que pertencem e na comunidade em que vivem ${ }^{(3,5,6)}$.

Infelizmente, por diversos fatores, nem sempre esses propósitos são alcançados, dentre eles está a inexistência de uma dinâmica eficiente na organização do trabalho e do atendimento prestado nos CAPS, que pode influenciar negativamente o fluxo dos serviços ${ }^{(7)}$. Desse modo, propostas de aplicação de estratégias e ferramentas de gestão para a organização do serviço são relevantes, não só para o campo da Saúde Mental, mas para a Saúde Coletiva, pois pode proporcionar melhoria na qualidade do cuidado prestado ${ }^{(8)}$.

Um exemplo de ferramenta que pode ser utilizada é o fluxograma. Estratégia útil para a otimização do atendimento, sua implantação possibilita uma visão nítida sobre os fluxos em curso no momento da produção do cuidado à saúde, permitindo a detecção de seus problemas ${ }^{(8,9)}$.

Assim, sugeriu-se, no presente estudo, a revisão do fluxograma de atendimento de um CAPS, devido ao fato desse seguimento, antes de ser fundamentado em conhecimento empírico, ter sido adquirido por meio de determinações que não contemplavam o itinerário que o paciente deveria adotar dentro do serviço, o que causava transtorno no atendimento para o paciente e para o serviço. Diante desse pressuposto, o presente trabalho tem por objetivo relatar a implantação de um fluxograma para os atendimentos de rotina em um Centro de Atenção Psicossocial.

\section{SÍNTESE DOS DADOS}

Trata-se de um relato de experiência de caráter intervencionista desenvolvido por acadêmicos do curso de Enfermagem, do Instituto de Ciências da Saúde, vinculado à Universidade da Integração Internacional da Lusofonia Afro-Brasileira - UNILAB, durante o estágio obrigatório da disciplina de Processo de Cuidado em Saúde Mental, realizado no mês de abril de 2016, em um Centro de Atenção Psicossocial (CAPS) localizado no município de Redenção, Ceará, Brasil.

O CAPS faz o acompanhamento de pessoas com transtornos mentais dos municípios da região na qual está inserido. No que se refere à rotina de atendimentos e ao público, está de acordo com a Portaria $\mathrm{n}^{\circ}$ 189/MS/03/2002 ${ }^{(10)}$, que constitui a 
demanda de atendimento do CAPS como tipo I, com serviço de atenção psicossocial nos dois turnos de atendimento e com atividades diárias em saúde mental e oficinas terapêuticas. São demandas do serviço estudado: depressão, ansiedade, psicoses, neuroses e retardo mental. Dispõe de uma equipe multiprofissional composta por um enfermeiro, um técnico de enfermagem, um médico psiquiatra, um psicopedagogo, um terapeuta ocupacional e um psicólogo.

Utilizou-se o método de observação participante e sistemática, pois busca a interação entre investigador e os grupos sociais $^{(11)}$. Utilizou-se também a técnica da roda de conversa, estratégia que proporciona a criação de canais de diálogo privilegiado para obter as informações necessárias para a construção da intervenção mediante participação das pessoas que usufruem do serviço ${ }^{(12)}$.

A observação sistemática do processo de trabalho da equipe do CAPS deu-se desde o acolhimento dos usuários até o momento da consulta com os profissionais de saúde. Após esse primeiro momento, puderam-se reconhecer as fortalezas encontradas, como o empenho dos trabalhadores e profissionais de saúde. Quanto às fragilidades, observou-se a sobrecarga de atendimentos decorrente do quantitativo insuficiente de profissionais. Esse mesmo problema repercute na organização do serviço, que acaba por não realizar a acolhida às pessoas usuárias, assim como também não realiza atividades em grupo, que são essenciais para o relacionamento terapêutico.

Observou-se o seguinte fluxo no atendimento pelo CAPS: os indivíduos com problemas psicossociais teriam que ter o encaminhamento de Unidade Básica de Saúde (UBS). Em seguida, fariam a triagem com a equipe multidisciplinar, em que seria indicado qual o grau de intervenção necessária, e, em seguida, teriam acompanhamentos mensais com um profissional específico considerando-se seu quadro clínico.

Nesse momento, um Projeto Terapêutico Singular (PTS), que visa criar um plano terapêutico de cuidados para o indivíduo, deve ser elaborado pela equipe multidisciplinar levando em consideração as necessidades, singularidades e desejos do usuário e família. Isso permitirá que a díade profissional-usuário crie vínculos e que haja o compartilhamento de saberes entre ambos. O profissional que o atende terá ainda a responsabilidade de acompanhar as pessoas durante todo o percurso da terapia naquele serviço, fornecendo a intervenção de outros profissionais ou serviços de apoio imprescindível, e, finalmente, garantindo a alta e o seguimento do tratamento em outro serviço ${ }^{(13)}$.

Já nos atendimentos subsequentes, observou-se que os usuários chegavam no dia da sua consulta já aprazada e apresentavamse na recepção para pegar a ficha de espera. Não havia nenhuma acolhida na entrada da instituição nesse momento. Em seguida, a atendente encaminhava-os para a espera do atendimento. É importante destacar que as pessoas esperavam na sala de espera da consulta sem haver qualquer forma de distração durante esse momento. A ambiência era inadequada, sendo esse espaço quase sem luminosidade. Em seguida, eram encaminhados para seu profissional específico, como psiquiatra, psicólogo, terapeuta ocupacional ou enfermeira. E, por fim, seguiam para seu domicílio. Na figura 1 pode-se observar como era o fluxo de atendimento no CAPS antes do novo fluxograma implementado.

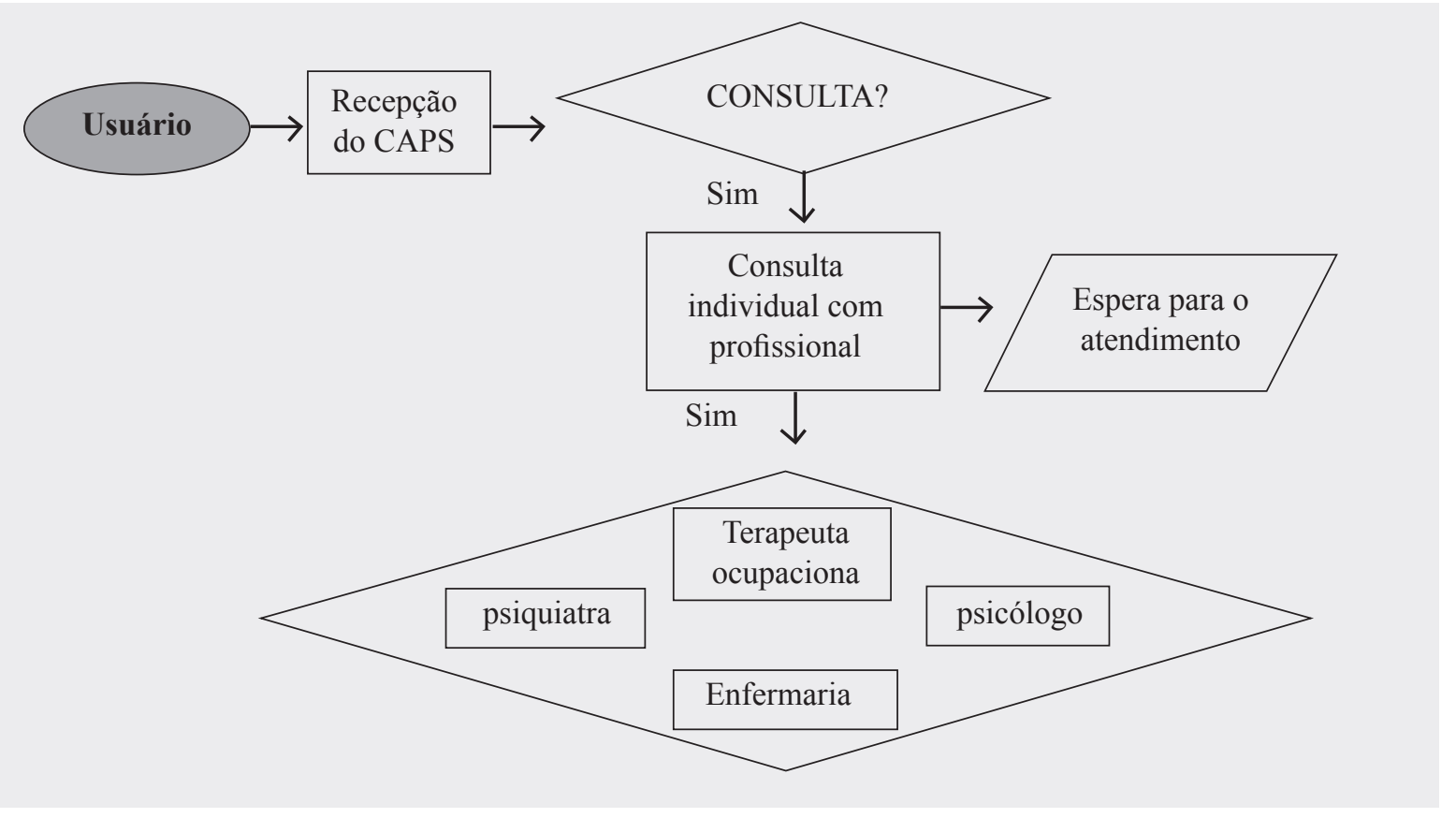

Figura 1 - Fluxo de atendimento no CAPS antes do novo fluxograma. Redenção, CE, 2016. 
Depois de realizadas as observações do fluxo da unidade, foi proposto um fluxograma alternativo, construído mediante a participação das pessoas usuárias que frequentavam o serviço, visando o conforto e o bem-estar dos mesmos enquanto aguardam seu atendimento.

Sentiu-se, dessa forma, a necessidade de ampliar o acolhimento ao usuário na chegada ao CAPS. Ressalta-se que o acolhimento é extremamente necessário, pois é o primeiro contato da pessoa usuária no momento da procura pelo atendimento, sendo um espaço para escuta terapêutica. Sendo assim, o profissional deve utilizar esse momento para conhecer a história de vida do indivíduo ${ }^{(14)}$.

Outro problema observado foi a ociosidade da pessoa usuária durante a espera pelo atendimento, que poderia se prolongar por horas. Havia ainda situações conflituosas entre os que aguardavam a consulta, em que alguns tentavam adentrar ao consultório antes dos demais. Todo esse âmbito gerava uma situação de estresse para todos.

Nessa perspectiva, problemas como a ociosidade dos usuários no período de espera da consulta puderam ser minimizados. Foi possível programar atividades, tais como rodas de conversa em sala de espera e realização do acolhimento logo na chegada para a consulta. Frisa-se que o acolhimento é indispensável em qualquer serviço de saúde, além de ser uma diretriz da Política Nacional de Humanização (PNH), pois implica na escuta direta das queixas da pessoa, reconhecendo que ela tem problemas e que devem ser solucionados ou mesmo amenizados por meio de redes de compartilhamento de saberes ${ }^{(14)}$.

Nesse contexto, acolhiam-se os usuários, recepcionando-os, respondendo possíveis dúvidas quanto ao atendimento, convidando-os a participarem das rodas de conversa, que outrora não existiam, e eram encaminhados para onde as atividades estivessem sendo desenvolvidas. Foram tratados temas solicitados pelos usuários nas rodas e que estavam bastante em discussão, como Influenza A, Dengue e Zika, entre outros temas sugeridos por eles.

As rodas tinham um número de 10 a 20 pessoas por grupo. Existia uma rotatividade dos participantes. Alguns iniciavam, mas saíam antes de terminar devido a sua ida à consulta. À medida que as atividades prosseguiam, outras pessoas inseriamse no grupo. Vale salientar que a roda era aberta para todos que quisessem participar. O objetivo principal dessa estratégia ocorreu principalmente por sua característica de permitir que os participantes expressem, concomitantemente, suas impressões, conceitos, opiniões e concepções sobre os temas sugeridos, assim como possibilitou discutir reflexivamente as manifestações apresentadas pelo grupo.

A principal limitação encontrada nessa experiência foi com relação ao ambiente propriamente dito para a realização das atividades; o calor do local e a indisponibilidade de algum equipamento para amenizá-lo; o barulho, pois o local onde se realizava o grupo dava acesso direto ao corredor; a restrição de amplitude do espaço; a indisponibilidade de cadeiras suficientes e o fato de ser um dos nossos primeiros contatos em integrar a saúde mental com a gestão do serviço, assim como a rotatividade dos usuários e o tempo limitado que os estudantes permaneceram no CAPS para executar as atividades.

Com base nas informações colhidas, foi possível a construção conjunta do novo fluxograma com apoio dos profissionais de saúde e gestores locais, e sua implementação na unidade nos dias subsequentes de estágio no CAPS. A partir disso, desenvolveram-se estratégias, como acolhimento e rodas de conversa na sala de espera.

A nova proposta de fluxograma correspondeu em colocar as pessoas usuárias do CAPS em atividades de grupo, instrumento valioso para criação de grupos de trabalho e educação em saúde, formados por usuários, em um espaço de diálogo e interação. Conforme as atividades eram realizadas, as pessoas eram convidadas a se dirigirem aos seus respectivos atendimentos, enquanto os demais permaneciam na atividade. Essa nova proposta de fluxograma agilizou a rotina do serviço da unidade e dinamizou o programa de atendimentos.

As atividades desenvolvidas dentro do CAPS colaboram para uma maior e melhor inserção das pessoas usuários na sociedade. Um exemplo disso foi um estudo realizado em Fortaleza, Ceará, em um CAPS do tipo II, onde uma associação comunitária que o coordenava realizava diversas atividades para os usuários, como horta comunitária, farmácia viva, cursinho pré-vestibular, arteterapia, entre outras. A partir dessas intervenções, resultados positivos foram alcançados, dentre eles uma maior interação do indivíduo atendido no CAPS com os que não fazem parte desse serviço ${ }^{(15)}$. 


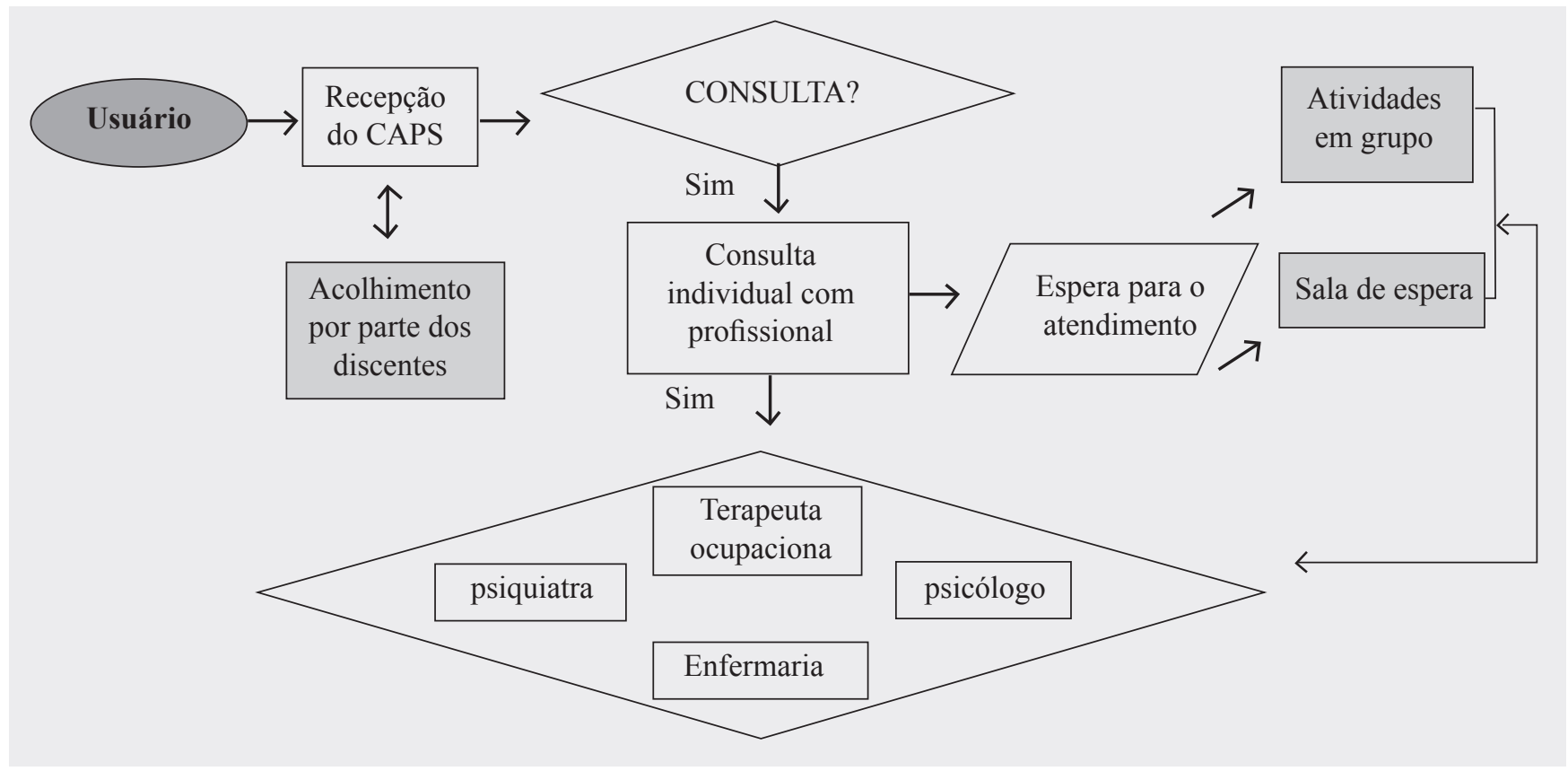

O novo fluxograma proposto se articulou conforme figura 2:

Figura 2 - Fluxo de atendimento no CAPS com novo fluxograma. Redenção, CE, 2016.

Após a implantação, constatou-se a sua importância para a melhoria na organização do processo de trabalho da unidade no que tange ao estabelecimento de uma rotina que vise prover acolhimento, oferecendo opções de atividades que possam ser realizadas durante a espera pelo atendimento.

Nesse sentido, pode-se afirmar que o uso de um fluxograma eficaz nas unidades de saúde e, nesse caso em especial, nos CAPS, se faz útil pelo fato de o mesmo ter a capacidade de proporcionar um olhar mais amplo dos processos de trabalho, possibilitando a identificação das áreas onde há os maiores obstáculos para o bom funcionamento da unidade, a fim de buscar soluções e melhores abordagens ${ }^{(16)}$.

Uma pesquisa realizada na cidade de São Paulo, São Paulo, Brasil, analisou a percepção de nove profissionais de saúde, dentre eles psicólogos, terapeutas ocupacionais, enfermeiros e psiquiatras que trabalhavam em um CAPS tipo III, acerca do acolhimento prestado aos usuários. Como resultado observou-se que o serviço tinha como principal estratégia o acolhimento. As práticas, segundo os relatos dos profissionais, se baseavam na escuta ativa e observação da singularidade de cada usuário, ou seja, havia a preocupação em realizar um trabalho humanizado, com a criação de vínculo e preocupação com o outro ${ }^{(17)}$.

Apesar da estrutura física não contribuir para o desenvolvimento das atividades, foi relatado pelas pessoas usuárias que foram úteis na minimização do estresse e da ansiedade. Na espera pelo grupo, pôde-se perceber como as pessoas usuárias ficavam tranquilas e participavam com compartilhamento das experiências singulares de cada um. Isso foi possível devido ao valor terapêutico para quem está participando, pois há uma maior interação entre usuários.

Assim, a reforma psiquiátrica tem sido relevante, uma vez que se baseia no conceito de desinstitucionalização. Sugere uma práxis, na qual os serviços devem configurar-se como uma rede de assistência, com foco na restauração da cidadania dos usuários atendendo suas demandas.

As limitações ocorreram devido ao pouco tempo que os estudantes permaneceram no CAPS para executar as atividades e perceber as reais mudanças com a implantação do fluxograma ou mesmo perceber se haviam mais fragilidades que poderiam ter sido pauta para mais intervenções. Quanto às fragilidades no serviço, observou-se a sobrecarga de atendimentos decorrente do quantitativo de profissionais insuficientes, repercutindo na organização. Entretanto, a universidade inserida na proposta de melhoria da qualidade proporciona crescimento para todos os envolvidos na promoção do cuidado humano e de qualidade.

Dessa forma, implantar um fluxograma traz ao CAPS organização, diminuição do nível de ansiedade pela espera do atendimento, eficiência no tratamento, melhor convívio profissional-usuário e, principalmente, provê bem-estar físico e mental para as pessoas ali presentes. Com as melhorias que o novo fluxograma pode trazer ao serviço, é necessário que haja mais estudos nessa temática para comprovar sua eficácia, visando adicioná-lo na rotina organizacional dos CAPS.

\section{CONCLUSÃO}

A presente experiência teve como base a construção de um novo fluxograma para organização do serviço de um CAPS, buscando-se deixar o ambiente harmonioso e acolhedor. 
Vivenciar a implantação de estratégias que proporcionem acolhimento a usuários dos serviços de saúde, principalmente a serviços que promovem o cuidado mental, no caso do CAPS, é uma ferramenta indispensável para a execução de um tratamento eficaz e eficiente. Tais estratégias devem seguir um fluxograma periódico, contínuo, harmonioso e diversificado que se enquadre ao público, mais especificamente, pessoas sob tratamento psicossocial.

\section{REFERÊNCIAS}

1. Souza ACS. Avaliação da estrutura e processos de trabalho em um Centro de Atenção Psicossocial [dissertação]. Goiânia: Universidade Federal de Goiás; 2015.

2. Carvalho MAP, Dias MD, Miranda FAN, Ferreira MO Filha. Contribuições da terapia comunitária integrativa para usuários dos Centros de Atenção Psicossocial (CAPS): do isolamento à sociabilidade libertadora. Cad Saúde Pública. 2013;29(10):2028-38.

3. Alves HMC, Dourado LBR, Côrtes VNQ. A influência dos vínculos organizacionais na consolidação dos Centros de Atenção Psicossociais. Ciênc Saúde Coletiva. 2013;18(10):2965-75.

4. Oliveira WF. Éticas em conflito: reforma psiquiátrica e lógica manicomial. Cad Bras Saúde Mental. 2009;1(2):49-61.

5. Queiroz MS, Delamuta LA. Saúde mental e trabalho interdisciplinar: a experiência do "Cândido Ferreira" em Campinas. Ciênc Saúde Coletiva. 2011;16(8):3603-12.

6. Pinto CAG, Coelho IB. Co-gestão do processo de trabalho e composição da agenda em uma equipe de atenção básica. In: Campos GWS, Guerrero AVP, organizadores. Manual de praticas de atenção básica: saúde ampliada e compartilhada. São Paulo: Hucitec; 2008. p. 323-45.

7. Miranda L, Oliveira TFK, Santos CBT. Estudo de uma rede de atenção psicossocial: paradoxos e efeitos da precariedade. Psicol Ciênc Prof. 2014;34(3):592-611

8. Scheffer G, Silva LG. Saúde mental, intersetorialidade e questão social: um estudo na ótica dos sujeitos. Serv Soc Soc. 2014;(118):366-93.

9. Souza AC, Guljor APF, Silva JLL. Refletindo sobre os Centros de Atenção Psicossocial. Av Enfermería. 2014;32(2):292-8

10. Ministério da Saúde (BR). Portaria no 189 de 20 de março de 2002. Brasília: Ministério da Saúde; 2002.

11. Correia MCB. A observação participante enquanto técnica de investigação. Pensar Enfermagem. 2009;13(2):30.6.

12. Melo $\mathrm{MCH}$, Cruz GC. Roda de conversa: uma proposta metodológica para a construção de um espaço de diálogo no ensino médio. Imagens da Educação. 2014;4(2):31-9.

13. Pinto DM, Jorge MSB, Pinto AGA, Vasconcelos MGF, Cavalvante CM, Flores AZT, et al. Projeto terapêutico singular na produção do cuidado integral: uma construção coletiva. Texto \& Contexto Enferm. 2011;20(3):493-502.

14. Jorge MSB, Diniz AM, Lima LL, Penha JC. Apoio matricial, projeto terapêutico singular e produção do cuidado em saúde mental. Texto \& Contexto Enferm. 2015;24(1):112-20.

15. Nascimento VF. Fluxograma de acesso e atendimento de enfermagem em unidade de saúde da família. Rev Elet Gest \& Saúde. 2013;4(01): 1922-7.

16. Andrade DAB, Bossi MLM. Qualidade do cuidado em dois centros de atenção psicossocial sob o olhar de usuários. Saúde Soc. 2015;24(3):887-900.

17. Godoy MGC, Viana APF, Vasconcelos KGD, Bonvini O. O compartilhamento do cuidado em saúde mental: uma experiência de cogestão de um centro de atenção psicossocial em fortaleza, CE, apoiada em abordagens psicossociais. Saúde Soc. 2012;21(Supl 1):152-63.

\section{Endereço para correspondência:}

Maria do Socorro Távora de Aquino

Universidade da Integração Internacional da Lusofonia Afro-Brasileira - UNILAB

Instituto Ciências da Saúde. Unidade Acadêmica dos Palmares

Rodovia CE 060-Km 51

CEP: 62785-000 - Acarape - CE - Brasil

E-mail: socorrotavora1@hotmail.com. 